\title{
UNA MIRADA ALTERNATIVA A LA MIGRACIÓN: EL VOLUNTARIADO JUVENIL COMO FORMA DE PARTICIPACIÓN SOCIAL
}

\author{
An Alternative Look at Migration: Youth Volunteering as Social Participation
}

\author{
Rafael Alonso Hernández-López \\ Claudia Janet Valverde-Hernández
}

Resumen: En este artículo se busca dar cuenta de un fenómeno que ocurre paralelamente a la migración en tránsito por México: la participación juvenil mediante voluntariado, en concreto en el Centro de Atención a Migrantes y Refugiados de la organización FM4 Paso Libre en Guadalajara, Jalisco. Para lograr ese propósito se ponen en contexto las dinámicas migratorias en la globalización, el desvanecimiento del Estado para acompañar estos procesos y su férrea presencia para generar dinámicas de selectividad y exclusión. Se concluye que, frente a las lógicas del Estado, los jóvenes hacen frente a las necesidades y circunstancias de las personas migrantes y refugiadas, lo que da lugar a creativas formas de participación social que reconceptualizan prácticas de caridad y beneficencia.

Palabras clave: migrantes, jóvenes, voluntariado, participación social, FM4 Paso Libre.

Abstract: This article seeks to give greater visibility to a phenomenon that occurs simultaneously with the migration of people in transit through Mexico: the participation of young people who volunteer at the Migrant and Refugee Center of FM4 Paso Libre in Guadalajara, Jalisco. To this end, we discuss the dynamics of migration in a context of globalization, the absence of any governmental support for the migratory phenomenon, and, in contrast, the selectivity and exclusion that arise from the government's overwhelming presence. We conclude that, given the government's rationale, young people have to assume responsibility for dealing with the needs and circumstances of migrants and refugees, which gives rise to creative forms of social participation that re-conceptualize the practices of charity.

Keywords: migrants, young people, volunteering, social participation, FM4 Paso Libre.

Rafael Alonso Hernández López. Doctoren Ciencias Sociales porelCentro de Investigaciones y Estudios Superiores en Antropología Social, Unidad Occidente, México. Coordinador General de Dignidad y Justicia en el Camino A.C. (FM4 Paso Libre), México, y de la Red de Documentación de las Organizaciones Defensoras de Migrantes (REDODEM), México. Temas de especialización: migración internacional en tránsito por México y refugio, políticas públicas y derechos humanos; migración interna, jornaleros agrícolas, mercados de trabajo y racismo. Correo electrónico: coordinacion@fm4pasolibre.org.

Claudia Janet Valverde Hernández. Licenciada en Relaciones Internacionales por el Instituto Tecnológico de Estudios Superiores de
Monterrey, México. Coordinadora del área de Vinculación de Dignidad y Justicia en el Camino A.C. (FM4 Paso Libre), México. Temas de especialización: migración internacional en tránsito por México y refugio, respuestas de los Estados y participación ciudadana. Correo electrónico: comunitario@fm4pasolibre.org.

Enviado a dictamen: 21 de noviembre de 2017 Aprobación: 25 de abril de 2018 Revisiones: 2 


\section{Introducción}

$\amalg$ fenómeno de la migración ha sido uno de los que, quizá, ha captado más atención por parte de gobiernos, academia y sociedad. Sus múltiples aristas y problemáticas han dejado en claro que, cuando su comprensión parece próxima, más distante se encuentra. Tradicionalmente el estudio de un fenómeno como el referido ha tenido un abordaje muy variado, y se han centrado los esfuerzos en entender y atender los impactos y efectos en los lugares de origen (Herrera, 2002; Durand, 2003, Pellegrino, 2003), tránsito (Martínez, Cobo y Narváez, 2015; Nájera, 2016) y destino (Castillo, 2000; Bermúdez, 2010) de los migrantes, casi siempre poniendo énfasis en las personas migrantes para tratar de comprender los motivos del desplazamiento, las modalidades de su tránsito, las condiciones de su inserción en el destino o quizá las de su retorno (Durand, 2004; Fernández, 2011). Muy poco se ha hablado de las dinámicas que genera de manera directa e indirecta en los espacios en los que acontece la migración (Cárdenas y Vázquez, 2014; Moreno y Niño, 2013). En el presente texto se pretende evidenciar un fenómeno alterno que se gestó a raíz del tránsito migratorio por México, el cual se ha fortalecido con el tiempo y ha generado un modelo especial de participación juvenil. Se trata, en este sentido, de visibilizar cómo la migración, con sus complejas dinámicas, posibilita la emergencia de acción de carácter social, que se traduce en un beneficio igualmente social al entorno inmediato y no inmediato en el que se suscriben dichas prácticas.

El caso que se presenta corresponde a la experiencia de la asociación civil conocida públicamente como FM4 Paso Libre, que desde el año 2009 realiza una labor de acompañamiento integral a personas migrantes y refugiadas en el occidente mexicano. Los hallazgos que aquí se presentan derivan de un esfuerzo de investigación-acción que la propia organización desarrolla en las áreas donde trabaja. En concreto, lo que se ofrece es la sistematización y análisis de un proceso de discusión colectiva que incluye la aplicación cotidiana de entrevistas a más de una centena de voluntarios y voluntarias antes o durante su servicio en la organización.

\section{Globalización, migración y el desvanecimiento del Estado}

La migración es un fenómeno dinámico y complejo que requiere de diversas lecturas en el marco de los cambios sociales, políticos y culturales producidos por la globalización, en la que se ha hecho más evidente la interconexión, el intercambio y la integración selectiva (Béjar y Rosales, 2005; Cuervo, 2006) de fenómenos a grados y niveles nunca vistos, donde el mundo imaginado y las perspectivas de vida son un constante flujo (Appadurai, 2001). Se trata de un ir y venir de objetos, símbolos y personas, con un intercambio de ideas y valores de los cuales algunos se incorporan, y otros conviven a la par, se fusionan o desaparecen. En este escenario, las realidades se vuelven diversas o, siguiendo a Bauman (2003), "líquidas", lo que pone en evidencia esa dinámica de fluidez, maleabilidad y fragmentación.

En la globalización, la migración se vuelve un asunto que trasciende gobiernos y forma parte de la agenda internacional, regional, nacional y local. Comprende una compleja serie de cambios a nivel individual, familiar y de comunidad que actúan juntos de manera orgánica (Massey et al., 1991: 11) y que además tienen causas y consecuencias en los ámbitos de la cultura, la economía, la política y la ecología, en la reconfiguración de identidades y en el género.

Si bien es cierto que la migración es un fenómeno común en la historia de la humanidad, actualmente, siguiendo las condiciones del capitalismo en su faceta neoliberal, ha tomado derroteros que denotan su enorme complejidad, pues se presentan en su forma más lacerante, obligada y forzada por el funcionamiento de un sistema cuya lógica es la desigualdad. Desde esa óptica, el "mito organizativo del capitalismo", como lo refiere el propio Wallerstein (1998), asociado fundamentalmente con la idea de progreso, está teniendo repercusiones severas y muy directas en la vida de los pueblos y de las personas. 
Frente al avance del capitalismo en sus múltiples facetas se observa un retroceso y desvanecimiento del poder del Estado con base en dos supuestos: la condena de este último como su mayor enemigo y su más peligrosa amenaza, y el ensalzamiento de las ventajas y rendimientos del mercado frente a los desastres y desmanes que propicia la intervención estatal en la economía (García de Vega, 2012: 33). Siguiendo los preceptos neoliberales que pregonan la vivencia radical de la libertad, el laissez faire, laissez passer de los fisiócratas, la persona migrante pasará de ser un sujeto dependiente y necesitado del Estado, a ser un individuo activo y autorresponsable, un empresario de sí. Esto se logrará mediante la promoción de una cultura empresarial de autosubsistencia, con arreglo a los valores de la iniciativa - competitividad, flexibilidad y autorresponsabilidad-, con lo que ha de hacerse cargo de prevenir sus infortunios contratando los servicios pertinentes en un mercado (Vázquez, 2005). El aparente deterioro de la capacidad de gestión y acción del Estado se hace evidente también en el debilitamiento de las instituciones sociales, la omisión de estadísticas oficiales y la falta de políticas públicas para la migración, lo que tiene impactos graves para las personas migrantes porque localmente son dejadas al abandono, bajo el argumento de que no son capaces de gestionar sus propias condiciones de vida, y en su proceso migratorio son discriminadas, explotadas, excluidas, violentadas e incluso desaparecidas, es decir, prácticamente invisibilizadas.

Las condiciones económicas, culturales, políticas y ambientales han creado un marco propicio para que la movilidad humana sea más bien una forma de desplazamiento de poblaciones pauperizadas y marginales, tradicionalmente oriundas del sur global, en la búsqueda de un espacio, por más precario que sea, en el norte global. Esta lógica de articulación norte-sur tiene un sentido principalmente económico que alude al hecho de que la integración mundial se produce preferencialmente entre los países más desarrollados, donde el sur tiene un significado próximo a la exclusión y el marginamiento (Cuervo, 2006: 39). Según un informe de la Organización Internacional para las
Migraciones de 2015 se estimaba que para dicho año en el mundo existían 740 millones de migrantes internos y 232 millones de migrantes internacionales.

De manera particular, en la región centroamericana y norteamericana la migración se ha convertido en un tema prioritario en la agenda de los Estados, y cabe aclarar que las evidencias apuntan a que quien lleva la batuta en el tema es Estados Unidos. La posición geográfica de México, al compartir frontera con ese país y con la región de Centroamérica, resulta medular en la estrategia política estadounidense sobre migración.

Para los fines del presente trabajo, se prioriza la modalidad de migración de tránsito por el territorio mexicano, representada por personas provenientes de Honduras, El Salvador y Guatemala principalmente. La Red de Documentación de Organizaciones Defensoras de Migrantes (REDODEM) ha identificado tres razones que la motivan: 1) el factor económico, el desempleo, los salarios bajos y el encarecimiento de la canasta básica; 2) el tema medioambiental, desastres hidrológicos, la degradación medioambiental y la reducción de recursos vitales; y 3) la violencia, particularmente el contexto generalizado de la misma, la persecución por el crimen organizado, o bien en su expresión intrafamiliar (REDODEM, 2015).

\section{Migración en tránsito por México, el rostro de una vulnerabilidad persistente}

Las personas migrantes en tránsito tradicionalmente utilizan el tren de carga llamado "La Bestia" como medio de transporte para trasladarse dentro del territorio mexicano. La población centroamericana ingresa a este país por distintos puntos en los estados de Chiapas y Tabasco, que colindan con Guatemala. Desde dichos puntos los migrantes acceden a las vías del tren, en aras de continuar su camino hacia el norte del país. La ruta ferroviaria del Pacífico o del occidente, espacio de este análisis, proviene del centro de la República, pasando por ciudades como Irapuato, en Guanajuato, y cruzando por Guadalajara, Jalisco, hasta llegar a Nogales, Sonora, Mexicali o Tijuana en Baja California Norte (ver Mapa l). 
El tránsito de migrantes por México ha sido ampliamente caracterizado. Destaca en especial el nivel de violencia que padecen estos, siendo los delitos más comunes que sufren el robo, la extorsión, lesiones y secuestro; quienes los cometen son integrantes de la delincuencia organizada (45.72\%) y las autoridades (41.51\%) (REDODEM, 2015). Estas últimas incrementaron su participación en un 21.35\% con respecto a 2014, destacando el contubernio de las policías federales, estatales y municipales (REDODEM, 2015: 60). En el informe "Situación de los derechos humanos en México" (Comisión Interamericana de Derechos Humanos, 2015) se expone que la migración irregular representa una de las principales fuentes de ingresos para el crimen organizado en México, "situación que se explica por los altos beneficios que genera ésta en comparación con el bajo nivel de riesgo y los altos niveles de impunidad que implican para estas organizaciones la comisión de delitos en contra de los migrantes" (2015: 45).

En este contexto, la labor de organizaciones de la sociedad civil y de defensores de derechos humanos es crucial para visibilizar el cotidiano acontecer del tránsito migratorio y presionar a los gobiernos, así como para concientizar a la población mexicana en torno a la innegable necesidad de abrir nuestras sociedades hacia la solidaridad y hospitalidad. La mayoría de las acciones de la sociedad civil — organizada y no organizada — se materializa en las denominadas casas del migrante y en centros de derechos humanos, donde las personas reciben ayuda desde apoyo humanitario básico, hasta asesorías médicas, jurídicas y psicológicas.

\section{FM4 Paso Libre, un modelo de atención a migrantes basado en la juventud}

FM4 Paso Libre es una organización de la sociedad civil sin fines de lucro, conformada por un grupo interdisciplinario de personas, en su mayoría jóvenes, que por medio de la experiencia directa con la realidad migratoria en otras zonas de la República decidieron emprender un proyecto de intervención integral en la Zona Metropolitana de Guadalajara (ZMG). Su misión es brindar, promover y defender un trato digno y justo que respete los derechos humanos de las personas migrantes y refugiadas desde el occidente de México mediante la atención integral, la inserción social, la investigación y la incidencia política y social.

Las primeras formas de participación se remontan al año 2007, cuando un grupo de estudiantes de distintas universidades de la ZMG regresó a la ciudad después de haber colaborado en diversas casas del migrante del país y decidió emprender un proyecto a favor de esta población que podría clasificarse en tres etapas que se desarrollan a continuación.

\section{A. Ímpetu por la ayuda solidaria (2007-2011)}

Esta etapa marca el comienzo del trabajo juvenil en favor de las personas migrantes. En 2007 el grupo mencionado comenzó a reunirse de manera periódica para discutir sobre la realidad de la migración en tránsito por su ciudad. Se planteó un contexto en que las condiciones socioestructurales llamaban a generar una respuesta ante la emergencia humanitaria. La discusión se centró en los derechos humanos como eje rector, el derecho a no migrar, el paso libre como opción para todas las personas en situación de movilidad humana, la solidaridad como valor para la transformación social, y la crítica a la estructura neoliberal y desigual.

Desde el inicio, el grupo de jóvenes reconoció la importancia de articularse con distintos actores de la sociedad civil para generar un camino digno y justo para la población migrante; por ello, en 2008 se inició la vinculación institucional con distintas escuelas de la ZMG y con redes que trabajaban el tema de migración. ${ }^{2}$

Conscientes de que el trabajo en pro de la población migrante tendría que superar la sola voluntad de hacerlo, en 2009 se constituyó legalmente la asociación con el nombre Dignidad y Justicia en el Camino A.C. (manteniendo su nombre público como FM4 Paso Libre), con la que se buscaba que el esfuerzo modificara la cotidiana vinculación que existía en el país entre migración, voluntariado, caridad y beneficencia, y para tratar de incidir en un cambio en las condiciones 
migratorias. Desde esa lógica comenzó a operar el voluntariado en la organización.

Fue así como el 10 de mayo de 2010 se abrió formalmente el Centro de Atención a Migrantes (CAM) con la intención de tener un punto de encuentro que garantizara la continuidad de la atención y favoreciera la prestación de más servicios — ropa, comida, llamadas telefónicas, medicamentos y baño con un periodo de atención de 3 a 4 horas diarias-. En ese momento, la naciente organización pasó de estar constituida por ocho personas, a contar con un grupo de 35 jóvenes voluntarios que se repartían el trabajo de apertura y operación del CAM, mientras que otros buscaban donaciones de insumos.

En los primeros dieciocho meses de operación del CAM se constató que existía mucha voluntad y disposición por parte de los jóvenes que se acercaban a realizar su voluntariado; no obstante, en ocasiones este tipo de apoyo era esporádico, sin un proceso formal y sin mucha continuidad, por lo que se planteó la necesidad de repensar el modelo y las formas de participación de los jóvenes en el CAM, y en general en FM4 Paso Libre.

\section{B. Proceso de conformación e institucionalización (2012-2014)}

Tras la evaluación anual de 2011 se detectó la importancia de abrir un proceso de planeación estratégica que tuvo lugar durante el año 2012 para definir el rumbo de la organización durante los siguientes cinco años. A raíz de esa planeación se delimitaron las áreas de atención y servicios, de vinculación, de investigación y de desarrollo institucional. El proceso de institucionalización, a su vez, originó un crecimiento en el número de voluntarios, así como en su formación.

El voluntariado pasó de 35 a 70 personas, y se logró conformar equipos de aproximadamente diez personas para la atención de lunes a sábado en el CAM. Este crecimiento fue posible gracias a un fuerte trabajo de difusión en escuelas, universidades e iglesias para conseguir que las personas se comprometieran en el servicio a migrantes. Asimismo, se crearon nuevas funciones al interior del voluntariado; por ejemplo, se creó la figura del encargado de día, persona responsable de dirigir, motivar y dar seguimiento al equipo durante la jornada. Esta estrategia contribuyó a una mayor cohesión entre los jóvenes, así como a aumentar el sentido de pertenencia y de integración. Cada equipo generó sus propias dinámicas de reflexión de la problemática y de contención frente a las complejas historias de migrantes que día a día se acompañaban.

La figura del encargado de día resulta medular para el proceso de seguimiento de las labores de la organización hasta la actualidad. En el perfil de este puesto se contempla que debe ser desempeñado por personas con experiencia en atención directa a la población migrante, con disposición para el trabajo en equipo y con habilidades para organizar y coordinar actividades, así como con conocimiento en materia de derechos humanos y migración. Es importante mencionar que toda persona que comienza a desempeñar esa función pasa por una experiencia mínima de voluntariado general de seis meses, tiempo en el que se va capacitando en diferentes temas que le serán de utilidad durante su servicio como encargado.

Durante el período 2012-2013 se formalizó la participación en diversas redes y colectivos de incidencia a nivel nacional e internacional. ${ }^{3}$ Asimismo, se llevaron a cabo labores de investigación, difusión y sensibilización del fenómeno migratorio, todas ellas con participación de voluntariado.

\section{Ampliación de incidencia política y acompañamiento integral (2015-2017)}

En la tercera etapa, que inició en 2015, se propició una interlocución más constante con autoridades gubernamentales, así como en espacios educativos y académicos, y se visibilizó el impacto de las políticas migratorias restrictivas mediante la colaboración y la publicación de diferentes textos de investigación ${ }^{4}$ en conjunto con otras organizaciones de la sociedad civil.

El año 2015 conllevó un gran reto dentro de la organización, cuando en julio, después de cinco años de funcionamiento, el CAM fue forzado a cerrar debido al clima de inseguridad y violencia prevaleciente en el estado de Jalisco. Fue importante el hecho 
de que, aun con el cierre, el voluntariado de FM4 Paso Libre no desistió en su labor humanitaria por sus sólidas convicciones, y se organizaron brigadas itinerantes en las vías del tren a fin de que las personas migrantes pudieran acceder a servicios básicos como alimentación y salud.

En octubre de 2015, luego de tres meses de intensiva incidencia con autoridades de los tres niveles de gobierno, el CAM reabrió sus puertas en un nuevo espacio físico, y pasó a convertirse, además, en un lugar de acogida para solicitantes de asilo. La reapertura del centro vino acompañada de una fuerte oposición vecinal que estaba basada en el temor y la desinformación. En una interesante dinámica de participación ciudadana, otro sector de la población local comenzó a declararse abiertamente a favor del proyecto y de la atención a migrantes en Guadalajara. De especial mención es el involucramiento de jóvenes, quienes a raíz del cierre del antiguo CAM se acercaron a la organización para colaborar y garantizar la subsistencia de la misma.

En ese nuevo centro se brindaban diez horas de atención al día, los siete días de la semana. Esta dinámica conllevó un proceso de reformulación en el voluntariado, de modo que se conformaron dos turnos al día - matutino y vespertino - y la figura de encargado de día pasó a ser de encargado de turno - en total eran catorce- Cada uno de los turnos estaba conformado, por lo menos, por un equipo de ocho voluntarios, un equipo de cocina y su respectivo encargado. A partir de ese momento, el equipo de voluntariado creció de 70 a 140 personas. Con tanta gente participando de manera activa, envuelta no sólo en la asistencia, sino en un proceso de formación, el voluntariado comenzó un nuevo camino y pasó de implicar un agregado de virtudes en las personas, a constituirse en un mecanismo de participación ciudadana activa.

La extensión de horario, con los esfuerzos de las áreas de Acompañamiento Integral y Atención Jurídica, permitió dar seguimiento más oportuno a la población migrante y refugiada. Se atendieron un total de once casos jurídicos - reconocimiento de la condición de refugio, trámite de visas por razones humanitarias, denuncias ante ministerios públicos, apoyo en regularización, etcétera- - y quince casos médicos - amputaciones, infecciones severas, fracturas, VIH-Sida, entre otros - , todo ello en el período que va de octubre de 2015 a diciembre de 2016. Esta situación se intensificó con la inauguración de un albergue en diciembre de 2016, al contabilizar un total de ochenta casos de acompañamiento integral y nueve derivaciones a hospitales públicos de Jalisco durante 2017.

Con conciencia de la importancia de incidir en el espacio político, en el año 2017 la organización generó, en conjunto con el diputado independiente Pedro Kumamoto, una propuesta de ley estatal en materia de migración denominada Ley de Hospitalidad para ser presentada ante el Congreso del Estado de Jalisco. Asimismo, miembros de la organización se incorporaron al Consejo Ciudadano del Instituto Nacional de Migración y al Consejo Consultivo del Instituto Jalisciense para Migrantes.

\section{Lo que los jóvenes hacen posible con su trabajo...}

Las acciones de ayuda humanitaria integral se materializan en el CAM, donde la población migrante y refugiada recibe los siguientes servicios: apoyo en vestimenta, tres comidas al día, llamadas telefónicas, servicio de regaderas y baño, paquete de higiene personal, albergue, atención médica, atención jurídica y acompañamiento psicosocial. Mediante la atención directa se ha brindado apoyo a más de 35000 personas en su paso y estadía por la ZMG desde el año 2010.

De 2011 a 2014 se observó cómo el número de personas atendidas en el CAM fue en aumento o se mantuvo. Los primeros seis meses de 2014 se registró un promedio de atención de 511 personas al mes, a partir de noviembre de 2014 se contempló un descenso significativo en el número de personas atendidas, situación que se evidenció aún más durante los primeros meses de 2015, cuando se atendió a un promedio de 230 personas (ver Gráfica l). Esta disminución se enmarca en la implementación del Plan Integral Frontera Sur (PIFS), a partir del cual se incrementaron las acciones de detención y deportación migratoria de extranjeros en México. De julio de 2013 a junio de 2014 el Instituto 
Nacional de Migración realizó un total de 97245 detenciones, mientras que de julio de 2014 a junio de 2015 el total fue de 168280 personas detenidas - 73\% de aumento- (Boggs, Knippen y Meyer, 2015). A pesar de la implementación del PIFS, las personas migrantes buscaron nuevas rutas para internarse y trasladarse a lo largo del país, por lo que repuntó el número de las atenciones en 2016 (ver Gráfica 2).

$\mathrm{El}$ corredor occidente se caracteriza por ser una ruta predominantemente de varones, ya que el 96\% de quienes transitan por ella son hombres, en su mayoría de nacionalidades de países centroamericanos. En la investigación de FM4 Paso Libre titulada Migración en tránsito por la Zona Metropolitana de Guadalajara (2013) se afirma que la población migrante procedía de los siguientes lugares de origen: $43 \%$ de Honduras, 30.2\% de México, 14.9\% de Guatemala, 9.6\% de El Salvador y $1.5 \%$ de Nicaragua. No obstante, desde el 2013 en años recientes se observa un incremento significativo en el porcentaje de personas hondureñas, al pasar de representar el 43\% en 2013, al 69\% en 2016, al tiempo que se generó un descenso en la población mexicana -30.2\% en 2013 y 16\% en 2016 - (ver Gráfica 3). El incremento de migrantes de origen hondureño refleja una tendencia regional de aumento de la migración centroamericana, a la par de un descenso en la migración mexicana hacia Estados Unidos. En Honduras, la inestabilidad política que generó la destitución del presidente Manuel Zelaya en 2009, el impacto de tormentas tropicales en 2011 y de huracanes como el Harvey en 2017, y el crecimiento desenfrenado de las maras y grupos criminales, así como la reelección del presidente Juan Orlando Hernández en 2017, crearon en ese país condiciones que explican el constante incremento de migración con destino a Estados Unidos y México.

En 2017 se registró un descenso en la atención brindada a migrantes en el CAM, de acuerdo con los testimonios de éstos últimos; tal situación fue producto de la incertidumbre que generó la elección del presidente Trump en Estados Unidos, de su discurso antimigrante, así como de las medidas que propuso para abordar la problemática de la migración, factores que sirvieron como freno temporal para la migración. Con base en la comunicación directa con personal de los albergues miembros de la REDODEM, durante los meses de febrero, marzo, abril y mayo de 2017 el flujo de migrantes descendió hasta en un 50\%. En el caso del CAM de Guadalajara, se observó que en los primeros meses de 2016 se atendió a un promedio de 566 migrantes por mes; no obstante, en el mismo período en 2017 fue de 321. Incluso en materia de detención, aunque no es concluyente el dato, se observó un cambio en el comportamiento de la tendencia, que puede corresponder con lo observado en los albergues del país. Mientras que en 2016 hubo 159872 eventos de deportación, en 2017 sólo se registraron 80353 (Unidad de Política Migratoria, 2018).

Respecto al perfil de las personas migrantes que recurren al CAM, de acuerdo con la información acumulada, la mayoría son jóvenes de entre 18 y 31 años de edad (54\%), es decir, migrantes en edad productiva para el mercado laboral, solteros (53.6\%), con poco o nulo acceso — seis años o menos- a educación escolarizada (52.5\%). La información obtenida permite afirmar que la ruta del Pacífico se caracteriza por un perfil de migrantes en su mayoría de nacionalidad hondureña, varones, jóvenes, de baja escolaridad, solteros, que tienen la intención de residir en Estados Unidos. No obstante, a partir de 2016 se observan otras tendencias: mayor flujo de niñas, niños y adolescentes acompañados y no acompañados; incremento en solicitantes de derecho de asilo y ascenso en el porcentaje de aquellos que ven en México su lugar de destino.

\section{La participación social de los jóvenes en el voluntariado con personas migrantes}

Cuando en otras regiones del mundo la incorporación voluntaria a organizaciones parece haber quedado atrás o está en entredicho por las altas cantidades de abandono (Vecina, Chacón y Sueiro, 2009), y en un marco de fuertes críticas hacia la juventud y sus actitudes por la aparente baja participación política y social de este sector, el proyecto de FM4 Paso Libre 
da muestra de una situación que, si bien es particular, puede dar luz sobre la participación social de jóvenes a través de la experiencia de voluntariado.

Siguiendo a Thompson y Toro (2000), un voluntario es considerado aquel que ofrece su tiempo y su talento a favor de una causa sin recibir remuneración ni pretender un lucro por ello. Si bien esta definición es limitada, tal como afirman los autores, es un punto de partida en el que se construye una práctica de participación que abarca una infinidad de situaciones que dan cuenta de un proceso social no homogéneo, identificable o medible de manera fácil; por el contrario, se trata de:

Un conglomerado no fácilmente clasificable de situaciones en las que los seres humanos demuestran, de disimiles maneras, su amor por la humanidad, su altruismo, su conciencia y responsabilidad política y social, sus pretensiones de ubicarse socialmente, o bien la única alternativa posible frente a situaciones como catástrofes o emergencias (Thompson y Toro, 2000: 1-2).

Ellos y ellas se enfrentan al gran desafío de relacionarse con una sociedad y un modelo económico que los seducen e incitan a consumir y a participar de los éxitos económicos, en un contexto donde, de manera simultánea, se les rechaza, excluye, ignora o castiga por su propia condición juvenil (Sandoval, 2000). Se encuentran, pues, en un fuego cruzado, en el que se suscitan fenómenos de gran impacto social como el de la migración.

La participación de los jóvenes en proyectos que llevan a cabo organizaciones sociales se vuelve un aspecto nodal en el ejercicio de su vida pública; aunque su dinámica de asociación a estas iniciativas responde a motivaciones muy variadas, sin duda refleja una voluntad de uso del tiempo libre de manera integradora (Sandoval, 2000):

Romper una rutina diaria de una burbuja o una zona de confort, abrirse a nuevos caminos, abrir la mente, pero dando la mano. Jamás me imaginé trabajar con un grupo de esta magnitud, me refiero a la vulnerabilidad y la grandeza de este grupo. Trabajar en este equipo es de un significado grande, significa unión, fortaleza, no cerrarse a oportunidades y tener ideología falsa; es tener esa oportunidad de crecer tanto personal, como social (Alberto, voluntario, 18 años, 2018).

Las dinámicas de pertenencia y apropiación del proyecto reflejan de una manera clara la sensibilización de un sector de la población que, además, con su activismo está generando formas de participación social muy importantes para un contexto como el de la ZMG que históricamente ha sido catalogado como apático o poco participativo, donde prima una clara posición ideológica religiosa y clasista que en muchas ocasiones raya en el racismo y fomenta la exclusión. Sin embargo, en Guadalajara residen y actúan personas de muy diferentes orígenes socioeconómicos, preferencias culturales, creencias religiosas y posiciones políticas. A pesar de que esta diversidad y pluralidad está incrementando progresivamente, la sociedad local también enfrenta dificultades para aceptar las divergencias existentes, lo que desencadena, incluso, expresiones y acciones intolerantes hacia lo otro o lo diferente (Ramírez y De la Torre, 2009).

Este escenario también da cuenta de un compromiso y solidaridad hacia la población migrante, pues los jóvenes que llegan a FM4 Paso Libre no sólo asisten o atienden a las personas migrantes, sino que también experimentan nuevas formas de relacionarse, de mirar el mundo, de entender e interpretar su realidad y, por lo mismo, de ampliar su contexto. Los jóvenes se asumen como individuos capaces de transformar su comunidad ante realidades adversas, en las que se aprecia una nula o pequeña presencia del Estado mexicano:

Creo que estar en un voluntariado como FM4 ayuda a cumplir con funciones que a veces ni el mismo gobierno o gobiernos hacen, que es el cuidado de minorías vulnerables. A veces la mejor manera de incidir en la sociedad es por medio de activismos y voluntariados que pongan un indicio de que las cosas se pueden hacer mejor (Ivón, voluntaria, 20 años, 2018). 
La participación del voluntariado implica un beneficio no sólo para el migrante que recibe su atención y compañía, sino para la sociedad de la ZMG en particular, y para el país en general, pues los jóvenes muestran un claro compromiso que, si bien es impulsado de manera indirecta por la organización, busca transformar mentalidades y abonar a la crítica, asî como a la generación de propuestas que ayuden a hacer frente a las convulsas circunstancias del entorno social. Si bien el amplio grupo de voluntarios y voluntarias es muy diverso, la mayoría son jóvenes que, en conjunto, con sus acciones proyectan un mensaje de solidaridad y hospitalidad:

Al contarle a las personas que conoces sobre todo lo que haces dentro del albergue y sobre la realidad que estas personas viven, ya estás haciendo un impacto, porque ya más personas saben sobre la labor del albergue y se dan cuenta de que la migración es una dinámica social que está presente en la ciudad y en la cotidianidad (Amanda Carolina, voluntaria, 18 años, 2018).

Desde 2010, un total de 619 personas -430 mujeres y 189 hombres - han realizado voluntariado en FM4 Paso Libre; su compromiso ha generado un impacto considerable tanto en la vida de las personas migrantes y refugiadas, como en la sociedad de la ZMG. La mayoría han sido de nacionalidad mexicana —507 personas — , y una parte de otros países — l12 personas-. El voluntariado extranjero procede en su mayoría de países europeos y americanos. No puede obviarse que se trata en su mayoría de jóvenes de un estrato social de clase media y media alta, lo cual es también un indicador importante para futuras reflexiones. Se menciona esto porque, aunque muchos de ellos y ellas laboran a la par que estudian, sus condiciones materiales son un tanto propicias, lo cual favorece que donen su tiempo y creatividad.

En ese sentido, a diferencia de las aproximaciones tradicionales según las cuales los actos de solidaridad fluyen de mejor manera en contextos paralelos, como la exclusión o la desigualdad, la participación de los jóvenes en FM4 Paso Libre evidencia una interacción y un ejercicio de colaboración sin caer en el extremo de la ingenua caridad o el asistencialismo. De hecho, la experiencia de la organización se ha dirigido a la búsqueda de formas más consistentes y duraderas de solidaridad y compromiso social, con una perspectiva transformadora que nace de la necesidad inmediata de las personas migrantes, pero que no acaba en ellas, sino que busca convertirse en una fuerza de cambio social y cultural de gran alcance.

La participación en FM4 Paso Libre da cuenta también de un cambio de valores por parte de los jóvenes, pues las utopías juveniles encuentran un espacio de maduración en el servicio voluntario (Benedit, 2000). Por otro lado, como se dijo en el epígrafe inicial, en este escenario de consumo y acumulación, la interacción con las personas migrantes genera cuestionamientos personales entre los voluntarios, tal como lo enunciara en su momento uno de los primeros voluntarios de la organización:

Cuando me siento ensalzado, muy fregón, que nada me falta, veo al migrante, platico con él y me ayuda a poner los pies en la tierra. Cuando me siento jodido, que todo va mal, que no valgo nada, veo al migrante, platico con él y me ayuda a ver que mis problemas no son tan complicados (Fabricio, voluntario, 22 años, 2012).

Desde otro ángulo, este cambio en la escala de valores se hace latente en la manera como se describe la propia organización, pues se asume a sí misma sin una definición religiosa, lo cual contrasta con más de cincuenta proyectos que existen en el país que dependen de la Iglesia o de grupos religiosos. Aunque el esfuerzo de FM4 y de los jóvenes que participan trasciende cualquier definición vinculada a alguna denominación religiosa, no obstante da cabida y favorece la convergencia de jóvenes religiosos en formación, quienes en interacción constante enriquecen sus propias concepciones. Situados en un entorno de consumo, intercambios y tecnologías, el CAM es un espacio de cohesión social que recupera y pone en el 
centro a las personas, y a partir de ese entendimiento se generan acciones de participación social para que el movimiento persista.

En el CAM, los jóvenes han sido capaces de dejar de lado sus diferencias religiosas, ideológicas, partidistas, de clase o profesionales, para converger en algo que se revela como urgente, inmediato e importante. Esta heterogeneidad describe la conformación de un tipo de activismo juvenil en el que convergen posicionamientos y la acción concreta en el espacio de atención. Esa acción se vuelve resultado de la suma de las interacciones sociales de los jóvenes, a nivel individual, pero sobre todo a nivel de grupos, donde las afinidades de participación son determinantes para las experiencias de asociación. Esa experiencia es atravesada por cuestiones generacionales que inciden en los códigos y prácticas compartidas por parte de los jóvenes dentro de los colectivos, y que a su vez determinan las posibilidades de articulación dentro de las redes de activismo (Ávalos, 2016: 20-22).

Por ello se habla también de identidades juveniles (Brito, 2002), pues mediante su participación social los jóvenes construyen relación con base en espacios de acción y mundos de vida (Krauskopf, 1998), cuestionando o imaginando alternativas novedosas que resignifiquen su contexto cotidiano y su estar en el mundo, lo cual, para el caso de la migración en tránsito por México, tiene mucho que ver con imaginar sociedades solidarias y hospitalarias, donde prevalezca el respeto y el reconocimiento a la diversidad, la lucha por la equidad y la erradicación de la desigualdad frente a un Estado que se repliega cada vez más o que, cuando actúa, lo hace de manera coercitiva, atentando contra las personas:

El voluntariado es una manera de hacer protesta ante la realidad injusta que se vive, y no sólo se ponen en práctica dinámicas dentro del albergue, sino que se manda un mensaje a la sociedad de no criminalizar ni revictimizar, ni ponerles muros invisibles a las personas migrantes, sino de apoyarlas en su camino en la medida de lo posible (Sara, voluntaria, 23 años, 2018).
En definitiva, la participación juvenil da cuenta de una búsqueda de espacios de rehumanización en la que es necesario recuperar las relaciones cara a cara, pero también resignificar la relación con el Estado. En la interacción cotidiana entre el voluntariado se dan una confluencia y una articulación que podrían dar lugar, en términos de Maffesoli (1988), a nuevas tribus, que posibilitan la sociabilidad y pertenencia a un grupo en el que existen compromiso e implicación afectiva que se entrecruzan, y a veces se oponen, pero que abonan indudablemente a recrear las identidades de quienes participan en estos esfuerzos.

Ahora bien, con la evidencia presentada al referir las dinámicas de participación juvenil en FM4 Paso Libre, se puede reconocer que, para el caso mexicano, los aportes y experiencias de organizaciones como la referida están configurando, con sus particulares aportes, un movimiento de carácter social en el que se produce una articulación local/global que contribuye a la conformación de un "nosotros" entre el tránsito de la indignación a la esperanza/acción, posible mediante la autorreflexividad de los participantes y su carácter no violento (Castells, 2012).

\section{Consideraciones finales}

En el contexto actual de globalización, las migraciones se generan en la complejidad. Mientras que en los lugares de origen se niega a las personas el acceso a bienes y servicios, por lo que se ven forzadas a desplazarse, en los lugares de tránsito se les violenta, son perseguidas y se busca a toda costa que sean deportadas. Estamos frente a un aparato estatal que delega en las personas la responsabilidad de hacer frente de manera absoluta tanto a sus necesidades, como a las circunstancias que en su vida acontezcan, posibilitando con ello la migración de miles de personas sin asumir una responsabilidad efectiva en dicho proceso. Por otro lado, ese mismo aparato estatal, en su expresión nacional, se encarga de contener, detener y deportar a quienes irrumpen en las fronteras de las geografías nacionales. 
Frente a esta bipolar actuación del aparato estatal mexicano desde hace más de una década, la sociedad civil ha hecho frente a las circunstancias derivadas de la migración, velando por el respeto y reconocimiento de los derechos humanos de las personas migrantes mediante acciones de tipo humanitario, así como de incidencia social o impulsando políticas e investigación, a fin de visibilizar lo que hasta hace poco tiempo era invisible.

De manera particular, se rescata el modelo de participación social que se lleva a cabo en FM4 Paso Libre, en el que son los jóvenes los protagonistas de estos novedosos procesos de interacción, reflexión, crítica y proposición; además de la ayuda que reciben las personas migrantes, la sociedad en la que están inmersos estos jóvenes también recibe un beneficio porque ellos contribuyen a crear conciencias dispuestas a plantear nuevos escenarios posibles, diferentes y alternos, en los que se sitúa en el centro a la humanidad, a las personas. Con su participación, los jóvenes dan cuenta de la posibilidad de conformación de relaciones sociales horizontales como base de una ciudadanía activa (Reguillo, 2008).

Es así como, desde el ángulo aquí propuesto, y en un contexto tan convulso, la migración, con toda su complejidad, se vuelve a la vez una posibilidad para repensar las maneras en las que nos hemos construido tradicionalmente, para sacar a flote lo mejor de las personas, y para poner en evidencia que también la migración enriquece a las sociedades y contribuye a la riqueza social, política y cultural. En el marco de participación descrito, se visualiza una tarea importante en relación con el análisis a fondo de estas identidades juveniles que se recrean en los espacios de atención a migrantes, ya que son el resultado de la intersección de la migración en tránsito, la retirada del Estado, la situación social de la ciudad y la participación de los jóvenes voluntarios.

\section{Notas}

${ }^{1}$ El nombre de la organización se eligió por analogía con las antiguas formas migratorias (FM) para regularizar la estancia de las personas extranjeras en el país, entre las cuales las más comunes eran la número 2 y la número 3. Conscientes de la alta vulnerabilidad y violencia hacia las personas migrantes, se optó por la creación imaginaria de la Forma Migratoria 4 (FM4) para velar por un "paso libre" de violencia, discriminación y criminalización, y garantizar un paso digno y justo por México.

2 Una de las primeras fue la Red Interinstitucional de Equidad de Género y Migración del Estado de Jalisco.

${ }^{3}$ Algunas de ellas son la Red de Documentación de las Organizaciones Defensoras de Migrantes (REDODEM) y el Colectivo para Incidir en el Plan Nacional de Desarrollo en el Tema de Migración, hoy conocido como Colectivo de Migraciones para las Américas.

4 "Derechos cautivos" y "Migrantes invisibles, violencia tangible".

\section{Referencias}

Appadurai, Arjun (2001). La modernidad desbordada. Buenos Aires: Fondo de Cultura Económica.

Ávalos G., Juan Manuel (2016). "Activismo político contemporáneo, acciones colectivas, jóvenes y tecnologías comunicativas en Guadalajara en Ixaya". En Revista Universitaria de Desarrollo Social, 11:11-31. México: Universidad de Guadalajara.

Bauman, Zigmunt (2003). Modernidad liquida. México: Fondo de Cultura Económica.

Béjar Navarro, Raúl y Héctor Rosales Ayala (2005). La identidad nacional mexicana como problema político y cultural. Nuevas miradas. México: CRIM-UNAM.

Benedit, René (2000). "Participación social y política de los jóvenesdela Unión Europea”. En Sergio Balardini (comp.), La participación social y política de los jóvenes en el horizonte del nuevo siglo. Buenos Aires: Consejo Latinoamericano de Ciencias Sociales. Disponible en: https://bit.ly/2KAW3xz (consultado el 27 de marzo de 2017).

Bermúdez Rico, Rosa Emilia. (2010). "Migración calificada e integración en las sociedades de destino/ Skilled Migration and Integration in Host Societies". En Sociedad y Economía. 19: 135-150. Disponible en: https:// bit.ly/2rPHVJX (consultado el 27 de marzo de 2017). 
Boggs, Clay, José Knippen y Maureen Meyer (2015). Un camino incierto. Justicia para delitos y violaciones a los derechos humanos contra personas migrantes y refugiados en México. México: Casa del Migrante de Saltillo Frontera con Justicia A.C., Fundar, Centro de Análisis e Investigación A.C., Albergue de Migrantes "Hermanos en el Camino", La 72, Hogar-Refugio para Personas Migrantes, Wola, Red Sonora y Un Mundo, Una Nación A.C.

Brito Lemus, Roberto (2002). "Identidades juveniles y praxis divergente; acerca de la conceptualización de la juventud". En Alfredo Nateras Domínguez (coord.), Jóvenes, culturas e identidades urbanas. México: Universidad Autónoma de México.

Cárdenas-Rodríguez, Rocío y Blanca Delia Vázquez Delgado (2014). "Recursos disponibles para la protección de mujeres migrantes en tránsito por Tamaulipas". En Papeles de Población, 20(79): 169-207. Disponible en: https://bit.ly/2rPInI9 (consultado el 27 de marzo de 2017)

Castells, Manuel Ángel (2012). Comunicación y poder. México: Siglo XXI Editores.

Castillo, Manuel Ángel (2000). "Las políticas hacia la migración centroamericana en países de origen, de destino y de tránsito". En Papeles de Población, 6(24): 133-157. Disponible en: https://bit.ly/2rOlUuG (consultado el 27 de marzo de 2018).

Comisión Interamericana de Derechos Humanos (2015). "Situación de los derechos humanos en México". S/1: la Comisión. Disponible en: https://bit.ly/2suvB2Q (consultado el 27 de marzo de 2018).

Cuervo, Luis Mauricio (2006). Globalización y territorio. Santiago de Chile: Instituto Latinoamericano y del Caribe de Planificación Económica y Social-Área de Gestión del Desarrollo Local y Regional, CEPAL. Disponible en: https://bit.ly/2INsgEx (consultado el 27 de marzo de 2018).

Durand, Jorge (2003). Clandestinos. Migración MéxicoEstados Unidos en los albores del siglo XXI. México: Universidad Autónoma de Zacatecas.

Durand, Jorge (2004). "Ensayo teórico sobre la emigración de retorno. El principio del rendimiento decreciente". En Cuadernos Geográficos, 35(.2): 103-116. Granada: Universidad de Granada.
García de Vega, P. (2012). "Neoliberalismo y Estado". En Pensamiento Constitucional, 4(4): 31-36.

Fernández Guzmán, Eduardo (2011). "Revisión bibliográfica sobre la migración de retorno". En Norteamérica, 6(1): 35-68. Disponible en: https://bit. ly/2IRqxOv (consultado el 27 de marzo de 2018).

FM4 Paso Libre (2013). Migración en tránsito por la Zona Metropolitana de Guadalajara: actores, retos y perspectivas desde la experiencia de FM4 Paso Libre. Guadalajara, México: FM4 Paso Libre.

FM4 Paso Libre (2016). El desafío del transitar-vivir en la ciudad para las personas migrantes en Guadalajara. Guadalajara, México: FM4 Paso Libre.

Herrera, Gioconda (2002). "La migración vista desde el lugar de origen”. En Iconos, 15. Ecuador. Disponible en: http://www.redalyc.org/articulo.oa?id=50901509 (consultado el 27 de marzo de 2018).

Krauskopf, Dina (1998). Participación y desarrollo social en la adolescencia. San José, Costa Rica: Fondo de Población de Naciones Unidas.

Maffesoli, M. (1988). Le déclin de l'individualisme dans les sociétés de masse. Paris: Méridiens Kliensieck.

Martínez, Graciela, Salvador David Cobo y Juan Carlos Narváez (2015). "Trazando rutas de la migración de tránsito irregular o no documentada por México". En Perfiles Latinoamericanos, 23(45): 127-155. Disponible en: https://bit.ly/2wPUWbh (consultado el 27 de marzo de 2018)

Massey, Douglas S., R. Alarcón, J. Durand y H. González (1991). Los ausentes. El proceso social de la migración internacional en el occidente de México. México: Alianza Editorial.

Moreno Mena, José Ascensión y Lya Niño Contreras (2013). "Una mirada hacia las organizaciones civiles de apoyo al migrante en Baja California y Sonora". En Región y Sociedad, 25(57): 61-96. Disponible en: https://bit.ly/2LbrBes (consultado el 27 de marzo de 2018).

Nájera Aguirre, Jéssica Natalia (2016). "El complejo estudio de la actual migración en tránsito por México: Actores, temáticas y circunstancias". En Migraciones Internacionales, 8(3): 255-266. Disponible en: https:// bit.ly/2Gqsj3W (consultado el 27 de marzo de 2018). 
Organización Internacional para las Migraciones (2015). Los migrantes y las ciudades: Nuevas colaboraciones para gestionar la movilidad. Ginebra: OIM.

Pellegrino, Adela (2003). La migración internacional en América Latina y el Caribe: tendencias y perfiles de los migrantes. Santiago de Chile: Centro Latinoamericano y Caribeño de Demografía (CELADE)-División de Población, Banco Interamericano de Desarrollo.

Ramírez Sáiz, Juan Manuel y Renée de la Torre Castellanos (2009). "El respeto a las creencias religiosas y la libertad de expresión artística: el caso de 'La Patrona' en Guadalajara”. En Espiral, 15(44): 199-25l. Disponible en: https://bit.ly/2L8K2Ag (consultado el 27 de marzo de 2018).

REDODEM (Red de Documentación de las Organizaciones Defensoras de Migrantes) (2015). Migración en tránsito por México: rostro de una crisis humanitaria internacional. México: autor.

Reguillo, Rossana (2008). Horizontes fragmentados. Comunicación, culturay pospolitica. Eldesorden globaly susfiguras. Guadalajara, México: Instituto Superior de Estudios de Occidente.

Sandoval, M. (2000). "La relación entre los cambios culturales de fines de siglo y la participación social y política de los jóvenes". En Sergio Balardini (comp.),
La participación social y política de los jóvenes en el horizonte del nuevo siglo. Buenos Aires: Consejo Latinoamericano de Ciencias Sociales, pp. 17-164. Disponible en: https://bit.ly/2KAW3xz (consultado el 27 de marzo de 2017).

Thompson, Andrés y Olga Lucía Toro (2000). "El voluntariado social en América Latina. Tendencias, influencias, espacios y lecciones aprendidas". En Revista Sociedad Civil, III(9). Disponible en: https:// bit.ly/2wTkzIg (consultado el 15 de enero de 2018). Unidad de Política Migratoria. Boletín estadístico 2018 Secretaría de Gobernación. Disponible en: https://bit. ly/1MphFr5_(consultado el 18 de enero de 2018).

Vázquez García, F. (2005). "Empresarios de nosotros mismos. Biopolítica, mercado y soberanía en la gubernamentalidad neoliberal". En Javier Ugarte Pérez (comp), La administración de la vida. Estudios biopolíticos. Barcelona: Anthropos.

Vecina Jiménez, M., F. Chacón Fuertes y M. Sueiro Abad (2009). "Satisfacción en el voluntariado: estructura interna y relación con la permanencia en las organizaciones". En Psicothema, 21(1): 112-117.

Wallerstein, Immanuel (1998). Impensar las ciencias sociales. México: Siglo XXI Editores. 
Gráfica 1. Migrantes atendidos por FM4 Paso Libre por mes y año, 2010-2017

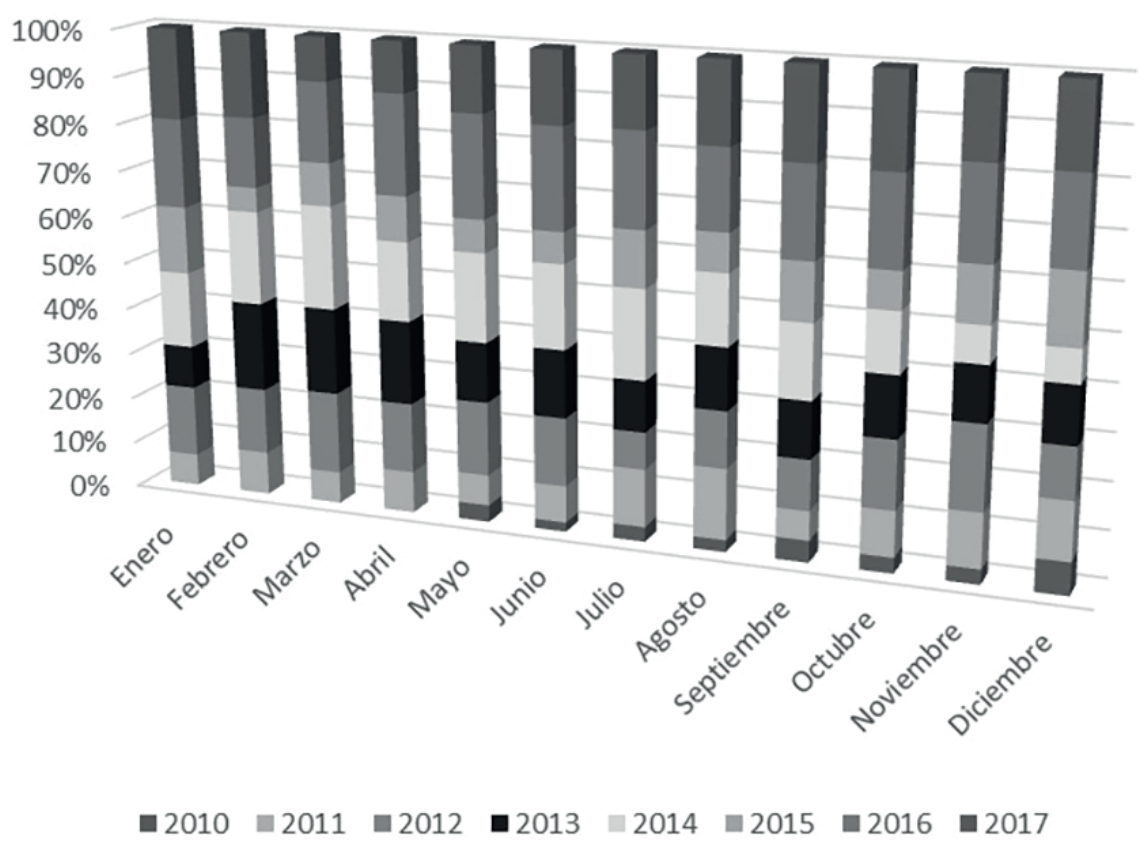

Fuente: elaboración propia con base en datos del archivo de FM4 Paso Libre.

Gráfica 2. Migrantes atendidos por FM4 Paso Libre. Totales anuales (2010-2017)

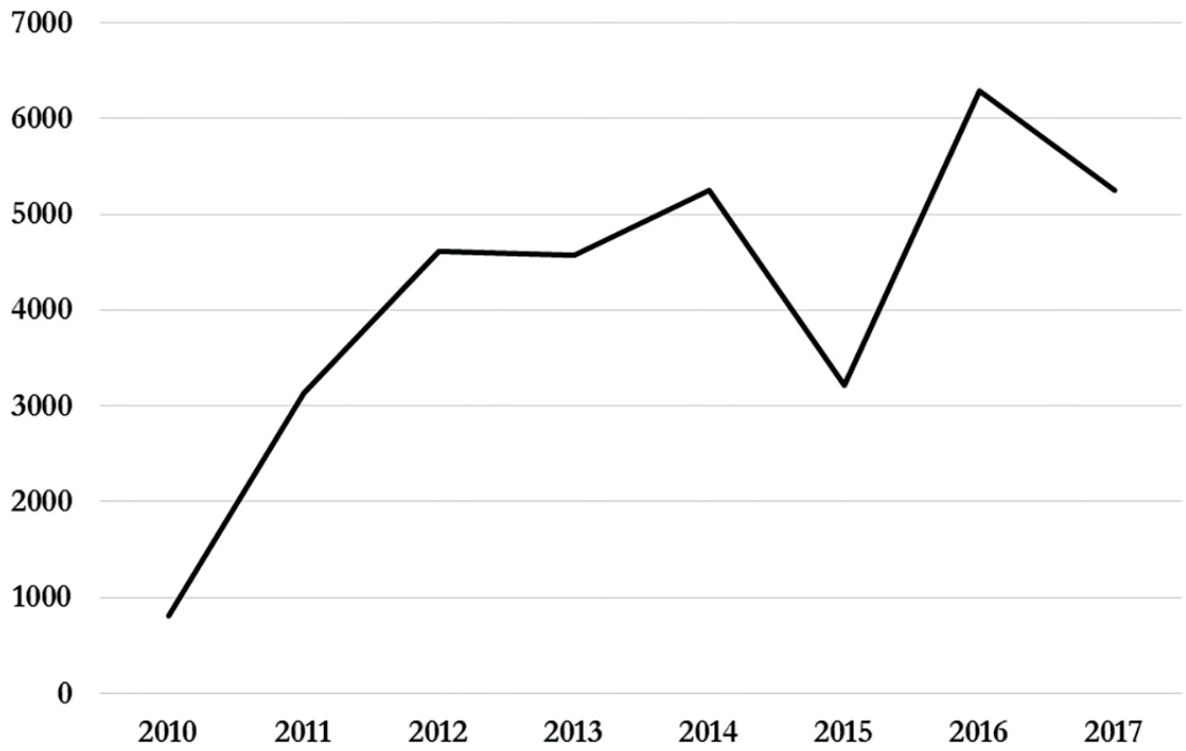

Fuente: elaboración propia con base en datos del archivo de FM4 Paso Libre. 
Gráfica 3. Nacionalidades de migrantes atendidos

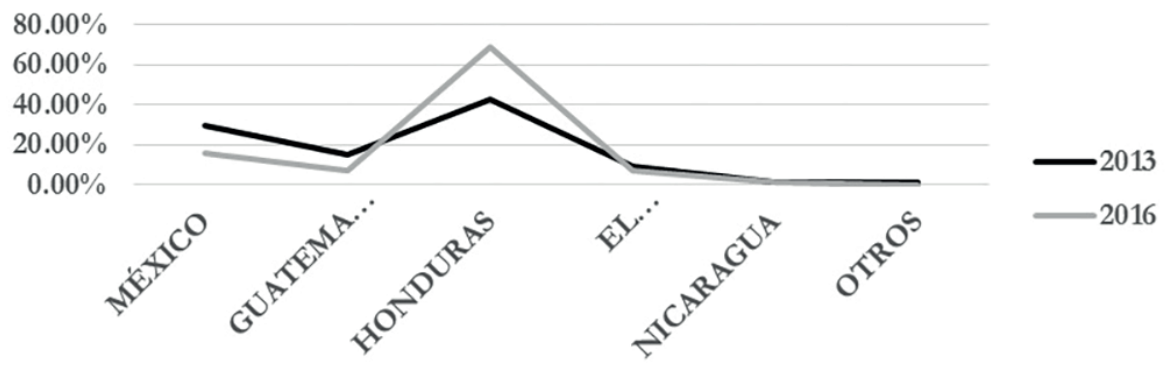

Fuente: elaboración propia con base en datos del archivo de FM4 Paso Libre.

Mapa 1. Corredores ferroviarios y puntos de apoyo para migrantes en México

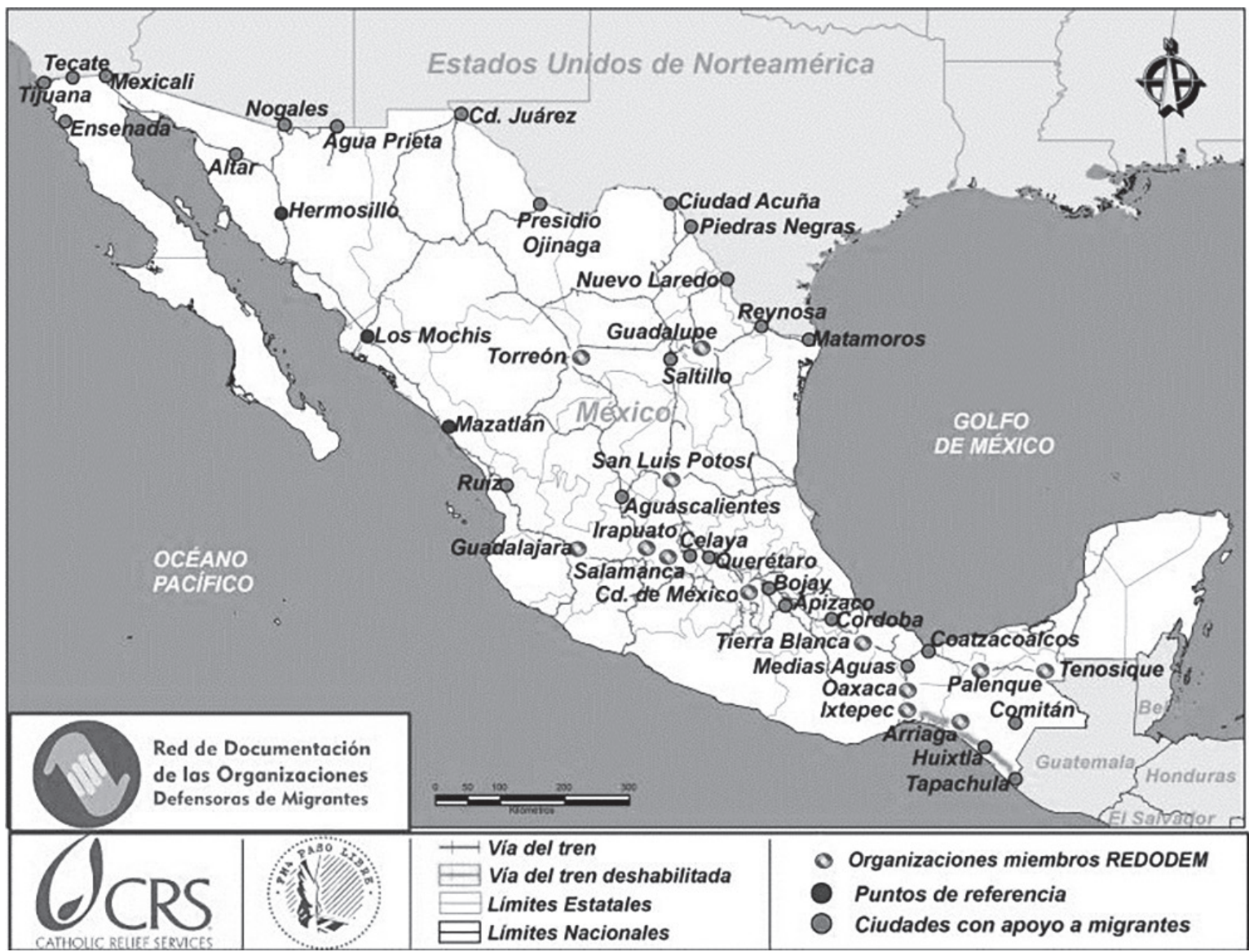

Fuente: FM4 Paso Libre (2013). Migración en tránsito por la Zona Metropolitana de Guadalajara: actores, retos perspectivas desde la experiencia de FM4 Paso Libre. 